\title{
Application of impedance boundary conditions to numerical solution of corrugated circular horns
}

Iskander, K; Shafai, L; Frandsen, Aksel; Hansen, Jesper

Published in:

I E E E Transactions on Antennas and Propagation

Link to article, DOI:

10.1109/TAP.1982.1142819

Publication date:

1982

Document Version

Publisher's PDF, also known as Version of record

Link back to DTU Orbit

Citation (APA):

Iskander, K., Shafai, L., Frandsen, A., \& Hansen, J. (1982). Application of impedance boundary conditions to numerical solution of corrugated circular horns. I E E E Transactions on Antennas and Propagation, 30(3), 366372. https://doi.org/10.1109/TAP.1982.1142819

\section{General rights}

Copyright and moral rights for the publications made accessible in the public portal are retained by the authors and/or other copyright owners and it is a condition of accessing publications that users recognise and abide by the legal requirements associated with these rights.

- Users may download and print one copy of any publication from the public portal for the purpose of private study or research.

- You may not further distribute the material or use it for any profit-making activity or commercial gain

- You may freely distribute the URL identifying the publication in the public portal 


\title{
Application of Impedance Boundary Conditions to Numerical Solution of Corrugated Circular Horns
}

\author{
KAMAL A. ISKANDER, LOTFOLLAH SHAFAI, SENIOR MEMBER, IEEE, AKSEL FRANDSEN, MEMBER, IEEE, AND \\ JESPER E. HANSEN, ASSOCIATE MEMBER, IEEE
}

\begin{abstract}
An integral equation method is used to formulate the problem of scattering by rotationally symmetric horn antennas. The excitation is assumed to be due to an infinitesimal dipole antenna, while the secondary field is obtained by assuming anisotropic impedance boundary conditions on the horn surface. This formulation is then used to investigate numerically the radiation from corrugated conical horns by approximating the corrugated surface with anisotropic surface impedances. The method is also used to study the scattering properties of receiver horns. In this case the external load is simulated by an impedance surface at the inner-end wall of the horn antenna, which is illuminated by an axially incident plane wave.
\end{abstract}

\section{INTRODUCTION}

$\mathbf{R}$ ECENTLY the problem of corrugated conical horns has been studied extensively in relation to the design of scalar feeds for reflector antennas. Generally, two main approaches have been utilized. The most elaborate approach is based on the modal expansion method, which has been used by many investigators to study the mode structure in corrugated waveguides and horns. Publication in this area is abundant; detail of the method and the results can be seen in the work of Clarricoats and Saha [1] or Jansen et al. [2]. The second method, which has not found widespread application, is based on a numerical solution of the corrugated horn problem [3].

In the modal expansion method, the fields both inside and outside the corrugated region are expressed in terms of appropriate mode functions. Boundary conditions on the corrugated surfaces are applied, and characteristic equations for determining the propagation constants of the modes are obtained. The solution of the characteristic equations gives the propagation constants and provides an understanding of the field distribution in the presence of the corrugations. For these reasons, the method enables one easily to obtain much important and practical information on corrugated horn antennas. It is, however, most useful for the cases where the corrugated surface is geometrically simple and coincides with a cylindrical or spherical coordinate surface.

In a numerical method, integral equations are usually used to determine the secondary fields from unknown induced currents on the corrugated surface. The method is general, in the sense that it can be applied to any arbitrary corrugated surface. However, since corrugations increase the surface area, an accurate solution of the problem requires large computer storage and computation time. In practice, therefore, it is usually not feasible to use the method to investigate any corrugated antenna with reasonable dimensions.

Manuscript received May 2, 1980; revised April 1, 1981. This work was supported by the Natural Sciences and Engineering Research Council of Canada and NATO Research Grant No. 1584.

K. A. Iskander and L. Shafai are with the Department of Electrical Engineering, The University of Manitoba, Winnipeg, MB, Canada.

A. Frandsen and J. E. Hansen are with the Electromagnetics Institute, Technical University of Denmark, Lyngby, Denmark.
In the present study we have used integral equations to formulate the problem of corrugated and rotationally symmetric horn antennas. However, to overcome the problem of extensive computer storage and computation time, we have represented the corrugations approximately with anisotropic surface impedances on the corrugated surface. With this representation, the boundary conditions are applied on a smooth surface just above the corrugations. Consequently, the large corrugated surface is reduced to a smaller one over the corrugations. The required surface impedances are determined approximately from the well-known relationships of the modal analysis.

Since only rotationally symmetric horns are considered, the integral equations for bodies of revolution are utilized [4]. The anisotropic surface impedances on the corrugated surface are used to obtain the integral equations, and the induced electric and the magnetic surface currents are expressed by proper Fourier expansions. The orthogonality of the azimuthal functions is used to obtain the integral equation of each azimuthal mode, which is then solved by approximating the mode currents in terms of triangular functions. To simplify the formulations, we used the method of Mautz and Harrington [4] to determine the coefficient matrices of the unknown currents. The matrix equations are solved, and the required secondary fields are determined by utilizing the standard equations.

The method is also used to study the scattering properties of receiver horns. In this case an impedance wall at the interior end of the horn is utilized to simulate the effect of an external load impedance. Boundary conditions are then applied to obtain the matrix equations and to determine the induced surface currents and the scattered fields in the usual manner.

In the next sections, the integral equations are obtained and are used together with the surface impedances to determine the required matrix equations. The computed radiation patterns of a $90^{\circ}$ corrugated conical horn are then compared with experimental data. Similarly the scattered field of a receiver horn matched to an external load is obtained and is compared with existing data.

\section{FORMULATION}

When a scattering surface supports the nonvanishing electric current $\bar{J}$ and the magnetic current $\bar{M}$, the scattered electric field may be obtained from [5] (with a suppressed time variation of $e^{j \omega t}$ )

$$
\bar{E}^{s}(\bar{P})=-j \omega \bar{A}-\nabla V-\nabla X \bar{F}
$$

where $\bar{A}$ and $\bar{F}$ are the electric and the magnetic vector potentials defined as

$$
\bar{A}=\mu \int_{s} \bar{J} \Phi d s^{\prime}, \quad \bar{J}=\hat{n} \times \bar{H}
$$




$$
\bar{F}=\int_{s} \bar{M} \Phi d s^{i}, \quad \bar{M}=\bar{E} \times \hat{n}
$$

and $V$ is the electric scalar potential given by

$$
V=\frac{1}{\epsilon} \int_{s} \sigma \Phi d s^{\prime}
$$

In these equations $\bar{J}$ and $\bar{M}$ are the electric and the magnetic surface current densities, $\sigma$ is the surface electric charge density, $\mu$ and $\epsilon$ are the permeability and the permittivity of free space, $s$ is the scattering surface, and $\Phi$ is the free-space Green's function of the form

$$
\Phi\left(\bar{r}, \bar{r}^{\prime}\right)=\frac{\exp \left(-j \kappa\left|\bar{r}-\bar{r}^{\prime}\right|\right)}{4 \pi\left|\bar{r}-\bar{r}^{\prime}\right|}
$$

where $\vec{r}$ and $\bar{r}^{\prime}$ are the positional vectors of the field and the source points, respectively, and $\kappa=2 \pi / \lambda$ is the wavenumber.

Now the electric charge density $\sigma$ can be substituted in terms of $\bar{J}$ by using the continuity equation which gives

$$
\sigma=-\frac{1}{j \omega} \nabla_{s} \cdot \bar{J}(\bar{r})
$$

where $\nabla_{s}$ is the surface divergence operator. Similarly, the magnetic current density $\bar{M}$ is related to $\bar{J}$ by the surface impedance and can be calculated from [6]

$$
\bar{M}=Z \hat{n} \times \bar{J}
$$

where $Z$ is the scalar surface impedance. This equations holds whenever the surface impedance is scalar. When the surface impedance is anisotropic, it can be presented by a dyadic $\overline{\bar{Z}}$. Equation (7) then takes the following form:

$$
\bar{M}=\overline{\bar{Z}} \cdot(\hat{n} \times \bar{J})=Z^{\phi} J^{\phi} \hat{U}_{t}-Z^{t} J^{t} \hat{U}_{\phi}
$$

where $Z^{t}$ and $Z_{-}^{\phi}$ are the components of the anisotropic surface impedance $\overline{\bar{Z}}$ along the $t$ and $\phi$ coordinates, respectively. In general, both $Z^{\tau}$ and $Z^{\phi}$ are the functions of the coordinate $t$ on the scatterer. Utilizing (2)-(8), one can write (1) in the form

$$
\begin{aligned}
\bar{E}^{\mathrm{sc}}(r)= & -j \omega \mu \int_{s} \bar{J} \Phi d s^{\prime}+\frac{1}{j \omega \epsilon} \nabla \int_{s}\left(\nabla_{s} \cdot \bar{J}\right) \Phi d s^{\prime} \\
& -\nabla x \int_{s} \overline{\bar{Z}} \cdot(\dot{n} \times \bar{J}) d s^{\prime}
\end{aligned}
$$

which involves only the surface current $\bar{J}$. When a field point approaches the scattering surface from the inside, the boundary condition $\vec{E}=\overline{0}$ is satisfied and for a field point on the surface gives

$$
\hat{n} \times \bar{E}^{\mathrm{inc}}=-\hat{n} \times \bar{E}^{\mathrm{sc}} .
$$

This equation together with (9) gives an integral equation for the unknown current $\bar{J}$. For bodies of revolution both the incident field $\vec{E}^{\text {inc }}$ and the induced current $\vec{J}$ can be expressed by Fourier series of the azimuthal coordinate. This approach provides an integral equation for each azimuthal mode, the solution of which gives the unknown mode current. This method is especially suited to horn antennas, since they normally operate in the dominant mode.

The evaluation of the mode currents may be carried out in a variety of ways. In earlier works [7] and [8], it was proposed to use the point matching method of Andreasen [9] to determine the mode currents. However, in a recent work [4] describing scattering from conducting bodies of revolution, Mautz and Harrington utilized the $E$-field and the $H$-field integral equations both in a separate and in a combined form to set up the required matrix equations. Comparing their formulation with (9), one notes that the first two terms of (9) are identical to the $E$-field integral equation and the third term has a form similar to the $H$-field integral equation. Thus, to simplify the process of obtaining the matrix equations and to benefit from their formulation, we have adopted this latter approach.

On a body of revolution the induced current $\bar{J}$ has components along two orthogonal surface vectors $\hat{t}$ and $\hat{\phi}$. According to the moment method each component can be represented by a finite set of expansion functions. Mautz and Harrington [4] have selected these expansion functions in the form

$$
\bar{J}_{n j}{ }^{t, \phi}=\hat{U}_{t, \phi} f_{j}(t) e^{j n \phi}
$$

and have reduced the integral equation to a matrix equation by using a set of testing functions

$$
\bar{W}_{m i}^{t, \phi}=\hat{U}_{t, \phi} f_{i}(t) e^{-j m \phi} .
$$

Utilizing a similar procedure, one can reduce (9) to a form

$$
\left[\begin{array}{cc}
{\left[Z_{m}{ }^{t t}\right]} & {\left[Z_{m}{ }^{t \phi}\right]} \\
{\left[Z_{m}{ }^{\phi t}\right]} & {\left[Z_{m}{ }^{\phi \phi}\right]}
\end{array}\right]\left[\begin{array}{l}
{\left[I_{m}{ }^{t}\right]} \\
{\left[I_{m}{ }^{\phi}\right]}
\end{array}\right]=\left[\begin{array}{l}
{\left[V_{m}{ }^{t}\right]} \\
{\left[V_{m}^{\phi}\right]}
\end{array}\right]
$$

where

$$
\begin{aligned}
& \left(V_{m}{ }^{t}\right)_{i}=\left\langle\bar{W}_{m i}{ }^{t}, E^{\text {inc }}\right\rangle \\
& \left(V_{m}^{\phi}\right)_{i}=\left\langle\bar{W}_{m i}{ }^{\phi}, \bar{E}^{\text {inc }}\right\rangle .
\end{aligned}
$$

$\left(\langle\bar{f}, \bar{g}\rangle\right.$ is the inner product $\int_{S} \bar{f} \cdot \bar{g} d s$, where $\bar{f}$ and $\bar{g}$ are tangential vectors on $s)$ and $\left[I_{m}{ }^{t}\right]$ and $\left[I_{m}^{\phi}\right]$ are column matrices of the unknown coefficients in the expansion of the current mode $m$. The four submatrices $\left[Z_{m}{ }^{t t}\right],\left[Z_{m}^{t \phi}\right],\left[Z_{m}{ }^{\phi t}\right]$, and $\left[Z_{m}{ }^{\phi \phi}\right]$ form what is known as the impedance matrix of the mode $m$. In the present problem each element of these submatrices contains the contribution of the last term of (9), due to the surface impedance. To relate the elements of our impedance matrix in (13) to those of [4], we may partition the above submatrices in the following form:

$$
\begin{aligned}
& {\left[Z_{m}{ }^{t t}\right]=\left[Z_{m}{ }^{t t, J}\right]+\left[Z_{m}{ }^{t t, M}\right]} \\
& {\left[Z_{m}{ }^{t \phi}\right]=\left[Z_{m}{ }^{t \phi, J}\right]+\left[Z_{m}{ }^{t \phi, M}\right]} \\
& {\left[Z_{m}^{\phi t}\right]=\left[Z_{m}^{\phi t, J}\right]+\left[Z_{m}^{\phi t, M}\right]} \\
& {\left[Z_{m}^{\phi \phi}\right]=\left[Z_{m}^{\phi \phi, J}\right]+\left[Z_{m}^{\phi \phi, M}\right]}
\end{aligned}
$$

where the superscripts $J$ and $M$ refer to the submatrices of the electric and the magnetic currents $\vec{J}$ and $\bar{M}$, respectively. In the above equation the submatrices $\left[Z_{m}{ }^{t t, J}\right],\left[Z_{m}{ }_{t \phi, J}\right]$, 
$\left[Z_{m}^{\phi t, J}\right]$ and $\left[Z_{m}^{\phi \phi, J}\right]$ are identical to the respective submatrices of the $E$-field integral equation in [4]. However, the elements of the submatrices of the magnetic current are related to those of the admittance matrices of the $H$-field integral equation in [4] by the following relationships:

$$
\begin{aligned}
& \left(Z_{m}{ }^{t t, M}\right) i j=-Z_{j}^{t}\left(Y_{m}^{\phi \phi}\right)_{i j} \\
& \left(Z_{m}^{\phi t, M}\right)_{i j}=Z_{j}^{t}\left(Y^{t \dot{\phi}}\right)_{i j} \\
& \left(Z_{m}^{t \phi, M}\right)_{i j}=Z_{j}^{\phi}\left(Y_{m}^{\phi t}\right)_{i j} \\
& \left(Z_{m}^{\phi \phi, M}\right)_{i j}=-Z_{j}^{\phi}\left(Y_{m}^{t t}\right)_{i j} .
\end{aligned}
$$

These relationships together with their evaluation from [4] complete the evaluation of the impedance matrix elements. It now remains to evaluate the elements of the excitation matrices $\left[V_{m}{ }^{t}\right]$ and $\left[V_{m}{ }^{\phi}\right]$ so that the induced currents $\left[I_{m}{ }^{t}\right]$ and $\left[I_{m}{ }^{\phi}\right]$ can be evaluated.

\section{THE EXCITATION MATRIX}

We assume that the field incident on the body of revolution is due to an $x$-directed electric dipole, located at a point $d\left(0,0, z_{d}\right)$. Thus the incident electric field may be obtained from

$$
\vec{E}^{\text {inc }}=-j \omega \bar{A}_{d}-\nabla \Phi_{d}
$$

where

$$
\bar{A}_{d}=\frac{-j \kappa \mu}{4 \pi} \bar{I} d l h_{0}{ }^{(2)}\left(\kappa\left|\bar{r}-\bar{r}_{d}\right|\right)
$$

and

$$
\Phi_{d}=\frac{\eta}{4 \pi} \bar{I} d l \cdot \nabla h_{0}{ }^{(2)}\left(\kappa\left|\bar{r}-\bar{r}_{d}\right|\right)
$$

where $\bar{I} d l$ is the moment of the dipole parallel to the $x$-direction, $\eta$ is the intrinsic impedance of free space, and $h_{0}{ }^{(2)}$ is the spherical Hankel function of the second kind. The required inner products may be shown to be

$$
\begin{aligned}
\left(V_{m}{ }^{t}\right)_{i}= & \left\langle\bar{W}_{m i}{ }^{t}, \bar{E}^{\mathrm{inc}}\right\rangle \\
= & \frac{\eta}{4 \pi}\left[-\kappa^{2} \int_{0}^{t_{i}} d t \rho f_{i}(t) \int_{0}^{2 \pi} d \phi\left(\bar{I} d l \cdot \hat{U}_{t}\right) h_{0}{ }^{(2)}\right. \\
& \cdot\left(\kappa\left|\bar{r}-\bar{r}_{d}\right|\right) e^{-j m \phi}+\int_{0}^{t_{i}} d t \frac{d}{d t}\left(\rho f_{i}(t)\right) \\
& \left.\cdot \int_{0}^{2 \pi} d \phi\left(\bar{I} d l \cdot \nabla h_{0}{ }^{(2)}\left(\kappa\left|\bar{r}-\bar{r}_{d}\right|\right)\right) e^{-j m \phi}\right]
\end{aligned}
$$

and

$$
\begin{aligned}
\left(V_{m}{ }^{\phi}\right)_{i}= & \left\langle\bar{W}_{m i}{ }^{\phi}, \bar{E}^{\mathbf{i n c}}\right\rangle \\
= & \frac{\eta}{4 \pi}\left[-\kappa^{2} \int_{0}^{t_{i}} d t \rho f_{i}(t) \int_{0}^{2 \pi} d \phi\left(\bar{I} d l \cdot \hat{U}_{\phi}\right)\right. \\
& \cdot h_{0}{ }^{(2)}\left(\kappa\left|\bar{r}-\bar{r}_{d}\right|\right) e^{-j m \phi} \\
& -j m \int_{0}^{t_{i}} d t f_{i}(t) \int_{0}^{2 \pi} d \phi\left(\bar{I} d l \cdot \nabla h_{0}{ }^{(2)}\right. \\
& \left.\cdot\left(\kappa\left|\bar{r}-\bar{r}_{d}\right|\right) e^{-j m \phi}\right]
\end{aligned}
$$

where $t_{i}$ is the length of the $i$ th generating curve. The evaluation of these inner products can be carried out in a manner similar to that used for the elements of the impedance matrix discussed in [4]. However, for an $x$-directed dipole the following relationships hold

$$
\begin{aligned}
& I \bar{d} l \cdot \hat{U}_{t}=I d l \sin \gamma \cos \phi \\
& I \bar{d} l \cdot \hat{U}_{\phi}=-I d l \sin \phi
\end{aligned}
$$

where $\gamma$ is the angle of the generating curve with the $z$-axis. Utilizing these expressions in (21) and (22) gives contributions only from $m= \pm 1$. Furthermore, when the basis functions are assumed to be in the form of triangular functions and are approximated by four impulses of the form [4]

$$
\begin{aligned}
& \rho f_{i}(t)=\frac{1}{\kappa} \sum_{p=1}^{4} T_{p^{\prime}} \delta\left(t-t_{i}^{\prime}\right) \\
& \frac{\partial}{\partial t}\left(\rho(t) f_{i}(t)\right)=\sum_{p=1}^{4} T_{p^{\prime}} \delta\left(t-t_{i}^{\prime}\right)
\end{aligned}
$$

where

$$
p^{\prime}=p+4(i-4) \text { and } i^{\prime}=p+2(i-1) .
$$

The elements of the excitation matrix (21) and (22) become

$$
\begin{aligned}
&\left(V_{m}{ }^{t}\right)_{i}=\left(\frac{-i \eta I d l}{4}\right) \sum_{p=1}^{4}\left[\frac{e^{-j \kappa R} d}{\kappa R_{d}}\right]_{i^{\prime}}\left\{T_{p}{ }^{\prime} \kappa \sin \gamma_{i}\right\} \\
&+\left[\frac{p_{i}^{\prime}}{R_{d}}\left(\frac{1}{R_{d}}+j \kappa\right)\right]_{i^{\prime}} T_{p^{\prime}}, \quad m= \pm 1 \\
& 0, \quad \text { otherwise } \\
&\left(V_{m}{ }^{\phi}\right)_{i}= \pm\left(\frac{\eta I d l}{4}\right) \sum_{p=1}^{4} \frac{1}{\kappa}\left(\frac{e^{j \kappa R_{d}}}{\kappa R_{d}}\right)_{i^{\prime}} \\
& \cdot\left[-\kappa^{2}+\left(\frac{1}{R_{d}}+j \kappa\right) \frac{1}{R_{d}}\right]_{i^{\prime}} T_{p}{ }^{\prime}, \quad m= \pm 1 \\
& 0, \quad \text { otherwise }
\end{aligned}
$$

where

$$
R_{d}=\left[\rho_{i^{\prime}}{ }^{2}+\left(z_{i^{\prime}}-z_{d}\right)^{2}\right]^{1 / 2}
$$

and $T$ and $T^{\prime}$ are the weighting factors of the impulse functions approximating the triangular functions and their derivatives.

\section{THE SCATTERED FIELD}

For a body of revolution with impedance boundary conditions the far scattered field in the direction $(\theta, \phi)$ may be written in the form

$$
\begin{aligned}
& E_{\theta} s=\frac{-j \omega \mu}{4 \pi r} e^{-j k r} F_{1}(\theta, \phi) \\
& E_{\phi} s=\frac{-j \omega \mu}{4 \pi r} e^{-j \kappa r} F_{2}(\theta, \phi)
\end{aligned}
$$


where

$$
\begin{aligned}
& F_{1}(\theta, \phi)=\int_{s}\left(\bar{J} \cdot \hat{U}_{\theta}+\frac{1}{\eta} \vec{M} \cdot \hat{U}_{\phi}\right) e^{j k \hat{r} \cdot \vec{r}^{\prime}} d s^{\prime} \\
& F_{2}(\theta, \phi)=\int_{s}\left(\bar{J} \cdot \hat{U}_{\phi}-\frac{1}{\eta} \bar{M} \cdot \hat{U}\right) e^{j k \hat{r} \cdot r^{\prime}} d s^{\prime}
\end{aligned}
$$

and the integrals are carried out over the scattering surface. The evaluation of these functions can be carried out in a manner similar to the elements of the excitation matrix and may be put in the form

$$
\begin{aligned}
& F_{1}(\theta, \phi)=\sum_{m=-M}^{M} \sum_{j=1}^{N}\left[\left(R_{m}^{t \theta}\right)_{j} I_{m j}{ }^{t}+\left(R_{m}^{\phi \theta}\right)_{j} I_{m j}{ }^{\phi}\right] \\
& F_{2}(\theta, \phi)=\sum_{n=-M}^{M} \sum_{j=1}^{N}\left[\left(R_{m}^{t \phi}\right)_{j} I_{m i}{ }^{t}+\left(R_{m}{ }^{\phi \phi}\right)_{j} I_{m j}{ }^{\phi}\right]
\end{aligned}
$$

where for a body of revolution with impedance boundary conditions one has

$$
\begin{aligned}
& \left(R_{m}{ }^{t \theta}\right) i=\sum_{q=1}^{4} C\left(m, q^{\prime}\right)\left[^{\cdot} j\left(J_{m+1}-J_{m-1}\right)\right. \\
& \left.\cdot\left(\sin \gamma_{i^{\prime}} \cos \theta-\frac{1}{\eta} Z_{i^{\prime}}{ }^{\prime}\right)-2 \cos \gamma_{i^{\prime}} \sin \theta J_{m}\right] \\
& \left(R_{m}^{\phi \theta}\right)_{i}=\sum_{q=1}^{4} C\left(m, q^{\prime}\right)\left(J_{m+1}+J_{m-1}\right) \\
& \cdot\left(-\cos \theta+\frac{1}{\eta} Z_{i^{\prime}}{ }^{\phi} \sin \gamma_{i^{\prime}}\right) \\
& \left(R_{m}^{t \phi}\right)_{i}=\sum_{q=1}^{4} C\left(m, q^{\prime}\right)\left(J_{m+1}+J_{m-1}\right) \\
& \cdot\left(\sin \gamma_{i^{\prime}}-\frac{1}{\eta} Z_{i^{\prime}}{ }^{t} \cos \theta\right) \\
& \left(R_{m}^{\phi \phi}\right)_{i}=\sum_{q=1}^{4} C\left(m, q^{\prime}\right)\left[j\left(J_{m+1}-J_{m-1}\right)\right. \\
& \cdot\left(1-\frac{Z_{i^{\prime}}^{\theta}}{\eta} \sin \gamma_{i^{\prime}} \cos \theta\right) \\
& \left.+\frac{2}{\eta} Z_{i^{\prime}}{ }^{\phi} \cos \gamma_{i^{\prime}} \sin \theta J_{m}\right]
\end{aligned}
$$

with

$$
J_{m}=J_{m}\left(\kappa \rho_{i^{\prime}} \sin \theta\right), \quad \text { Bessel function of order } m
$$

and

$$
\begin{aligned}
& C\left(m, q^{\prime}\right)=\pi j^{m} e^{j m \phi} e^{j \kappa z_{i} \cos \theta} T_{q^{\prime}} \\
& q^{\prime}=q+4 i-1 \\
& i^{\prime}=q+2 i-2 .
\end{aligned}
$$

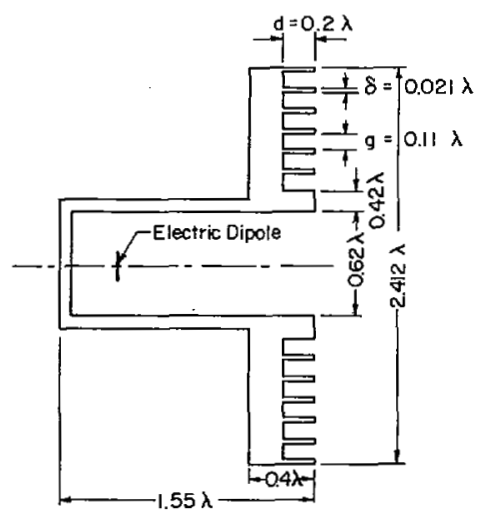

Fig. 1. A $90^{\circ}$ corrugated circular horn.

This sequence of equations completes the derivation of the scattered field for a body of revolution with impedance boundary conditions.

\section{APPLICATIONS}

\section{A. Corrugated Horns}

In this section we have applied the above method to investigate the radiation characteristics of a $90^{\circ}$ corrugated horn. The geometry of the horn is shown in Fig. 1. The excitation is due to an $x$-directed electric dipole which is located inside the horn, a quarter of a guide wavelength from the inner-end wall. As was pointed out in Section III, since this form of excitation contributes terms only from $m= \pm 1$ it is an ideal excitation for a horn with a transverse electric $\left(\mathrm{TE}_{11}\right)$ exciting mode.

To generate the elements of the impedance matrix we require a suitable equation for the surface impedance to represent the corrugations. From the modal analysis of [1] such a simple equation for the resonant corrugations can be shown to be

$$
\begin{aligned}
& Z^{\phi}=0 \\
& Z^{t}=-j \eta \tan \kappa d
\end{aligned}
$$

where $d$ is the depth of the corrugation. The validity of such a representation for nonresonant corrugations was also shown recently [10]. However, it was found that for nonresonant corrugations the surface impedance $Z^{t}$ must be averaged over the solid and corrugated portions of the surface. In other words, (38) must be modified to

$$
Z^{t}=-\frac{j g}{g+\delta} \eta \tan k d
$$

where $g$ and $\delta$ are defined in Fig. 1 .

To apply the matrix equation (13) to the present problem, we divide the inner and the outer generating curves of the horn into small segments and represent the current distributions over each segment by triangular functions. The resulting integrals are evaluated by approximating the triangular functions with four impulses [4]. Boundary conditions are applied which satisfy the surface impedances given in (37) and (39) over the corrugated portion of the horn. The resulting matrix equations is solved to give the surface current, which is used in (33) and (34) to find the $E$-plane and the $H$-plane radiation patterns. For different numbers of triangular functions, Fig. 2 compares computational and experimental results. The accu- 


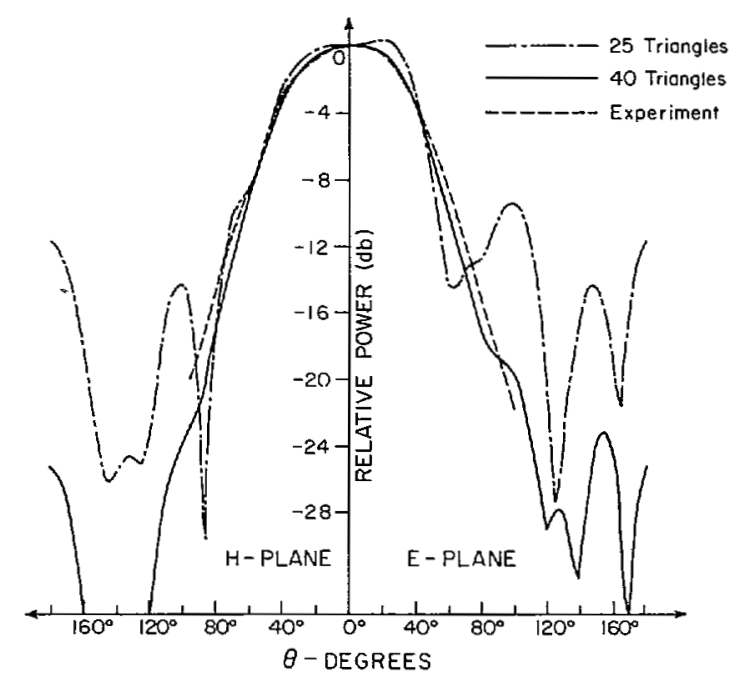

Fig. 2. Computed copolar patterns of corrugated horn of Fig. 1.

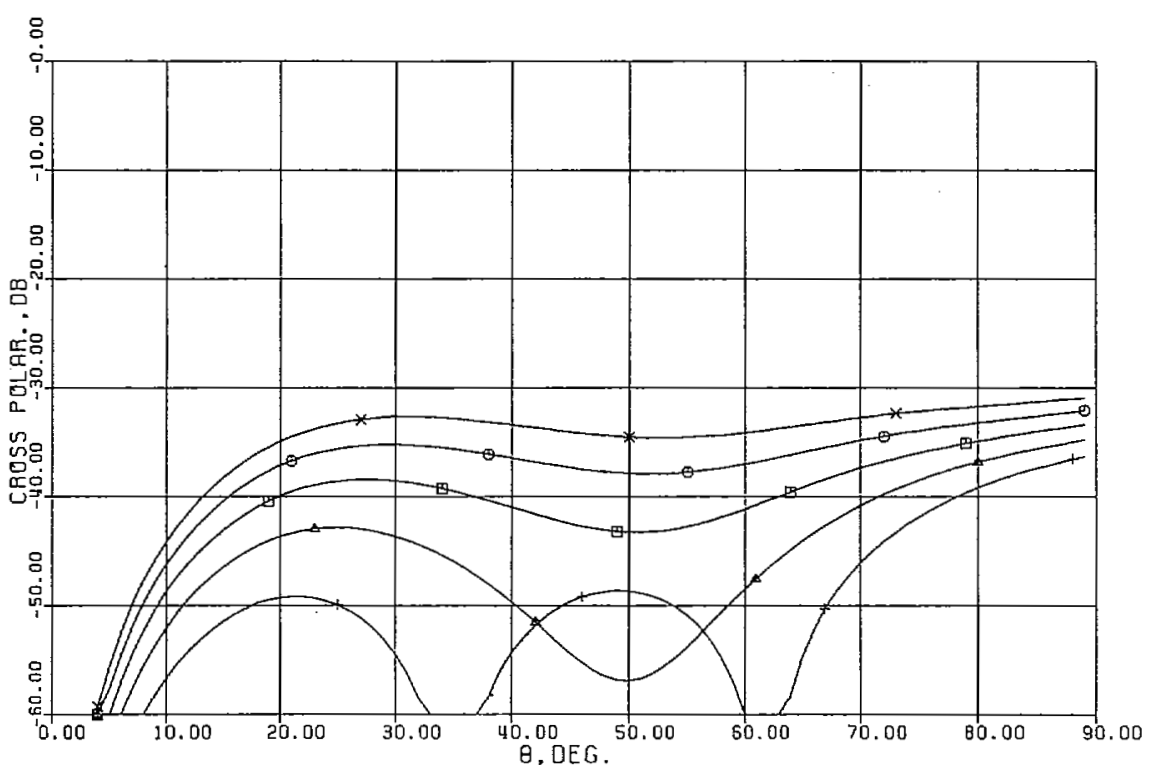

Fig. 3. Computed cross-polar patterns of the horn, shown in Fig. 1, for different corrugation depths near $d=0.15 \lambda x-\mathrm{x}$ $d=0.13 \lambda, \diamond-\circ d=0.14 \lambda, \square-\square d=0.15 \lambda, \Delta-\Delta d=0.16 \lambda,+-+d=0.17 \lambda$.

racy of the computation improves as the number of triangular functions increases to 40 ; beyond that point the improvement in the computed results is too small to justify additional computation time. If the approximate representation of the corrugations with an impedance surface is taken into account, the agreement between the two sets of results seems reasonable.

For the same geometry the computed cross-polarized radiations are shown in Fig. 3. For the geometry of Fig. 1 the cross-polarized radiation is generally high. However, as the results indicate, with small modification of the corrugation geometry, the level of cross-polarization can be reduced considerably. Note that in practice such an optimization can be carried out quite efficiently, since for a given horn geometry one needs only to modify the corrugation impedance and the elements of the coefficient matrix need not be evaluated for each new case. Unfortunately, we did not have experimental data to compare with the computed results for the crosspolarized radiation.

Our computations were carried out on an Amdahl 470/V7 using 60 triangle functions to represent the induced cur- rent, and required about $30 \mathrm{~s}$ central processing unit (CPU) time. The main part of this computation time was used, of course, to generate the elements of the impedance matrix and to solve the matrix equation for the surface current. The required computer time for calculating the field patterns is normally negligible.

Attempts were also made to reduce computation time by neglecting a portion of horn exterior wall. It was found, however, that no matter what fraction of the exterior wall is neglected the computed patterns never correspond to the experimental ones. Unfortunately, we have not found a satisfactory explanation for this behavior. In these cases, the generating contour does not form a closed surface, which is an inherent assumption in the development of the theory. Similar results were experienced by Frandsen [11].

\section{B. Receiver Horns}

In many practical cases it is desirable to study the scattering properties of receiver horns. However, because receiver horns are usually connected to a matched external load (with 
a matched horn we understand a horn terminated in a reflection free impedance $(\Gamma=0)$ and not a conjugate matched horn, where the load is the conjugate of the antenna impedance), the signal received by the horn does not radiate back. For this reason a direct solution of scattering by the horn does not correspond to the scattering of a horn under operating conditions. To overcome this problem Frandsen [11] has used a movable short in the. waveguide section of the horn to simulate the effect of the external load. In this paper we have solved the problem by using the impedance boundary condition. To be specific, the effect of the external load is simulated by impedance boundary conditions on the inner surface of the horn end wall. In particular, for a dominant mode horn the effect of an externally matched load may be simulated by an impedance surface on the inner end wall in the form of the mode impedance. For the $\mathrm{TE}_{11}$ mode propagating in the waveguide section of the horn, the wave impedances are given by [5]

$$
\begin{aligned}
& Z^{t}=\frac{E_{\rho}}{H_{\phi}}=\frac{\omega \mu}{\kappa_{z}} \\
& Z^{\phi}=-\frac{E_{\phi}}{H_{\rho}}=\frac{\omega \mu}{\kappa_{z}}
\end{aligned}
$$

where $\kappa_{z}$ is the axial propagation constant of the $\mathrm{TE}_{11}$ mode. Using these surface impedances on the horn interior end wall, we can evaluate the impedance matrix to give the required surface currents on a receiver horn. This method was used to study the scattered field of horns and waveguides with different external load impedances, and the results were compared with those of Frandsen. In all cases the agreement was found to be within the range of the computational errors.

Fig. 4 shows the geometry of a small conical horn, a socalled optimum horn. The horn is illuminated axially from the front by a plane incident wave generated by an electric dipole. The computed $E$ - and $H$-plane patterns are shown in Fig. 5 , which are compared with those of Frandsen. A single precision computation was used, and 60 triangular functions were utilized to represent the currents over the interior and the exterior walls of the horn. The agreement between the results is satisfactory, especially in the $E$-plane. In the $H$-plane the main discrepancy occurs around the $90^{\circ}$ off-axis points; otherwise the agreement is good. Because we have again used 60 triangular functions, the total CPU time for solution of the matrix equation and computation of the patterns was about 30 s.

\section{CONCLUSION}

A matrix equation for investigation of bodies of revolution with impedance boundary conditions was developed using the electric and magnetic scalar and vector potentials. Surface currents were approximated by triangular basis functions and the formulation was selected to be similar to that for conducting bodies of revolution. It was shown that the impedance matrix may be partitioned into two matrices such that one of the matrices is identical to the impedance matrix of conducting objects for the $E$-field integral equation. The elements of the other matrix are identical to the elements of the admittance matrix of conducting objects for the $H$-field integral equation, but are multiplied by the respective surface impedances.

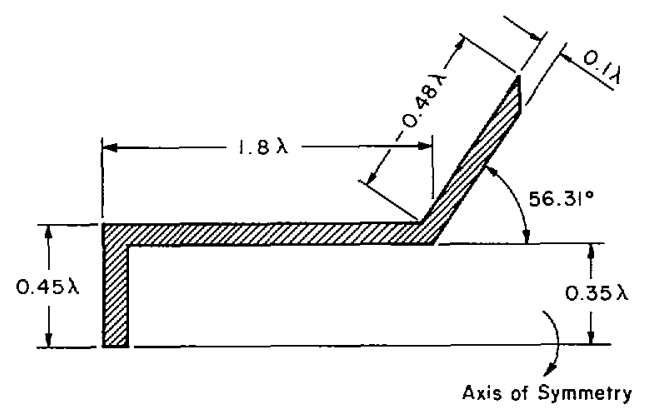

Fig. 4. Geometry of a matched conical horn.

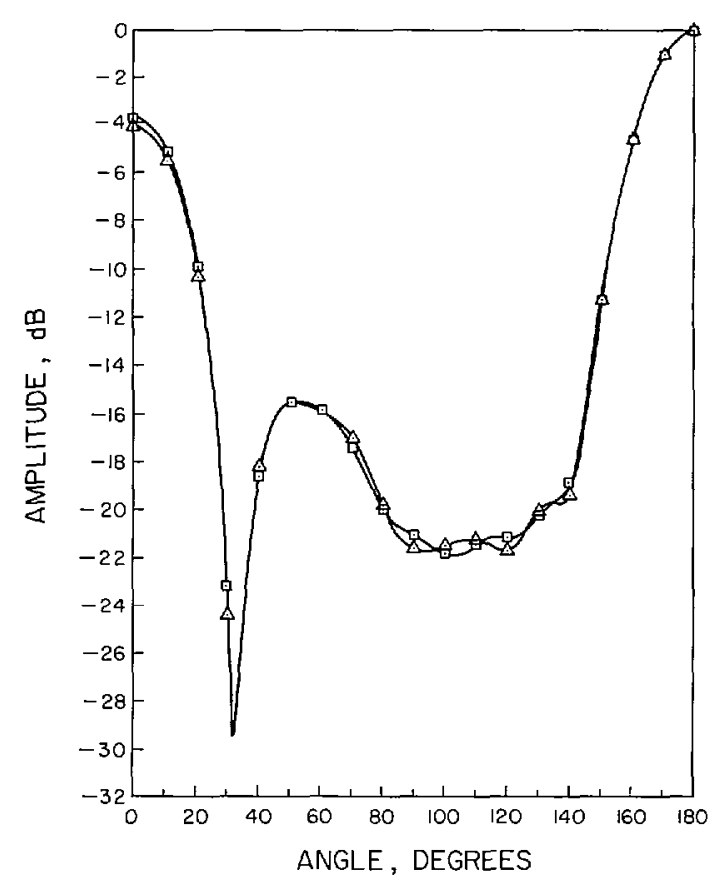

(a)

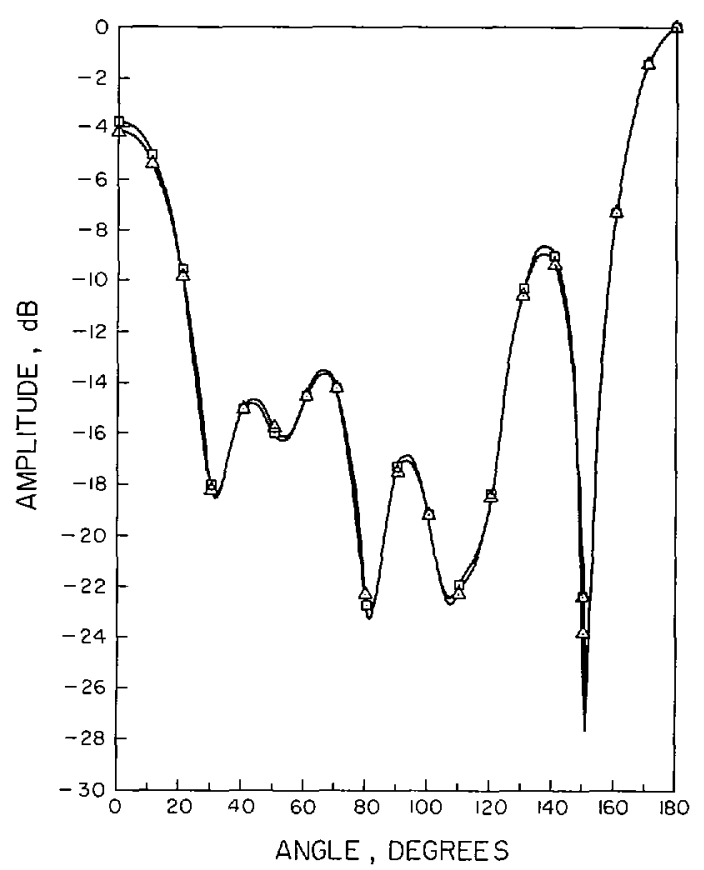

(b)

Fig. 5. Scattering patterns of the matched horn shown in Fig. 4. $\Delta$ Frandsen, $\square$ present work. (a) $E$-plane. (b) $H$-plane. 
The method was then applied to study the radiation properties of corrugated horns. Corrugations were approximated by anisotropic surface impedances, and the surface currents were then obtained for an electric dipole excitation. The computed copolarized patterns were compared with experimental data. The same method was also used to study the scattering from receiver horns. The effect of the external load impedance was simulated by an isotropic surface impedance on the interior end wall of the horn.

For investigating the properties of corrugated horns with impedance boundary conditions, this method reduces the large surface area of corrugations to a small surface over the corrugation. Since the required computation time is small, practical corrugated horns can be analyzed with relative ease. The method also reduces the computation time for investigation of receiver horns. The method can be similarly applied to other corrugated geometries, but care must be taken to model the corrugation properly.

\section{REFERENCES}

[1] P. J. B. Clarricoats and P. K. Saha, "Propagation and radiation behavior of corrugated feeds," Part I and II, Proc. Inst. Elec. Eng., vol. 118A, pp. I167-1186, 1971.

[2] J. K. M. Jansen, E. J. Jeuken, and C. W. Lambrechtse, "'The scalar feed," Arch. Electron Ubertragungstech., vol. 26, pp. 22-30, 1972.

[3] C. A. Mentzer and L. Peters, "Properties of cut off corrugated surfaces for corrugated horn design," IEEE Trans. Antennas Propagat., vol. AP-22, pp. 191-196, 1974.

[4] J. R. Mautz and R. F. Harrington, " $H$-field, $E$-field and combined field solutions for bodies of revolution," Univ. Syracuse. Tech. Rep. TR-77-2, 1977.

[5] R. F. Harrington, Time Harmonic Electromagnetic Fields. New York: McGraw-Hill, 1961.

[6] R. Mittra, Ed., Computer Techniques for Electromagnetics. Oxford: Pergamon, 1973.

[7] L. Shafai and J. E. Hansen, "Matrix formulation of corrugated feeds by using impedance boundary conditions," Electron. Lett., vol. 13 , no. 11, pp. $310-311,1977$.

[8] — "Correction to matrix formulation of corrugated feeds by using impedance boundary conditions," Electron. Lett., vol. 13, p. $491,1977$.

[9] M. G. Andreasen, "Scattering from bodies of revolution," IEEE Trans. Antennas Propagat., vol. AP-13, pp. 303-310, 1965.

[10] L. Shafai, K. A. Iskander, and J. E. Hansen, "Investigation of scattering by corrugated structures using impedance boundary conditions," Electron. Lett., vol. 14, no. 5, pp. 144-146, 1978.

[11] A. Frandsen, "Computation of scattering and radiation from open ended waveguides and small horns," presented at the IEEE G-AP Symp., Seattle, WA, June 1979.

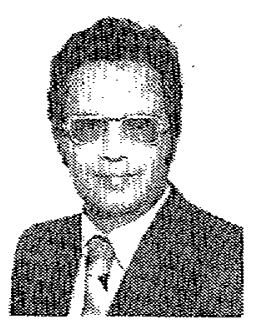

Kamal A. Iskander was born in Cairo, Egypt, in October 1945. He received the B.Sc. and M.Sc. degrees in electrical engineering from the University of Alexandria, Egypt, in 1968 and 1972, respectively, and the Ph.D. degree in Electrical Engineering from the University of Manitoba, MB, Canada, in 1980.

From 1968 to 1972 , he was employed as a Research Engineer and Lecturer in the Communications and Antennas Laboratory of the Faculty of Enginecring, University of Alexan-

dria. At the end of 1972, he joined the Electromagnetics Group, the University of Manitoba as Research Assistant. In 1979, he joined the Antennas Group at SPAR Aerospace Ltd, in Ste. Anne de Bellevue, PQ, Canada, where he worked on the development of Dichroic surfaces for earth stations and spacecrafts antennas. There he was also involved in the development phase of the antennas for the Anik-C and Anik-D Canadian communications satellites. His research interests are in applied electromagnetics, antennas design and measurements, and the application of numerical techniques to field problems.

Lotfollah Shafai (S'67-M'69-SM'75), for a photograph and biography please see page 667 of the September 1980 issue of this TRANSACTIONS.

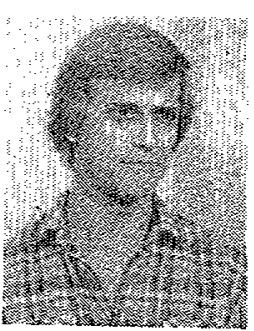

measurements.
Aksel Frandsen (S'77-M'80) was born in Denmark on November 5, 1953. He received the M.Sc.E.E. degree from the Electromagnetics Institute, Technical University of Denmark, Lyngby, Denmark, in 1978.

From 1978 to 1980 he carried out his Ph.D. study on numerical calculation of radiation and scattering from horn antennas using moment methods. In August 1980 he joined TICRA A/S where he has been engaged in computer simulation studies of inaccuracies in near-field

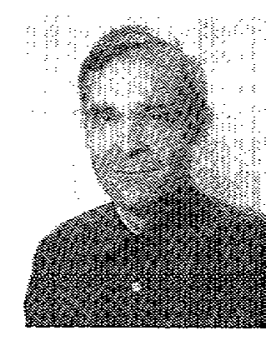
Electrical Engineering and Computer Sciences, University of California, Berkeley, as Postdoctoral Scholar. He has worked in the areas of antenna arrays and numerical methods in antenna design. Currently, he is responsible for the research program associated with the spherical near field antenna test facility, developed jointly by the European Space Agency and the Technical University of Denmark.
Jesper E. Hansen ( $\left.A^{\prime} 62\right)$ was born in Frederiksberg, Denmark, on November 14, 1929. He received the M.Sc. and $\mathrm{Ph} . \mathrm{D}$. degrees in electrical engineering from the Technical University of Denmark, Lyngby, Denmark, in 1957 and 1965 , respectively.

Since 1959 he has been with the electromagnetics Institute, Technical University of Denmark, where he is an Associate professor. During 1965-66 he was with the Electronics Research Laboratory and the Department of 